\title{
Ciencia básica y ciencia aplicada
}

\author{
Ruy Pérez-Tamayo, M.C. ${ }^{(1)}$
}

\section{Pérez-Tamayo $\mathbf{R}$. \\ Ciencia básica y ciencia aplicada \\ Salud Publica Mex 2001;43:368-372. \\ El texto completo en inglés de este artículo está disponible en: http://www.insp.mx/salud/index.html}

\section{Resumen}

En el contexto de una intervención en el Foro de Consulta Democrática de la Coordinación de los Institutos Nacionales de Salud sobre Enseñanza e Investigación en Salud, realizado en el Instituto Nacional de Cardiología Ignacio Chávez, se hace una revisión crítica de la clasificación convencional de la ciencia en básica y aplicada; además, se analiza lo que deberían ser la enseñanza y la investigación en salud. Para apoyar su propia clasificación de la ciencia en "bien hecha", la cual "genera conocimientos verificables sobre la realidad" y la "mal hecha", improductiva o productora de "puras mentiras" y "no debe patrocinarse", el autor revisa las definiciones utilitaristas y peyorativas como las que establecen ciencia comprometida y ciencia pura, ciencia útil y ciencia inútil, y ciencia práctica y ciencia esotérica, como sinónimos de ciencia aplicada y ciencia básica y afirma que, en México, esta diferenciación "para lo único que ha servido en el pasado es para justificar la reducción en el apoyo oficial a la ciencia básica, porque no estaba dirigida a resolver 'los problemas nacionales'o porque no caía en las prioridades establecidas en ese sexenio". En cuanto a educación e investigación en salud reconoce que el programa actual de formación de investigadores tiene una eficiencia muy baja y propone un estudio científico crítico, realizado por un equipo de especialistas, interdisciplinario, "para integrar la carrera del investigador científico desde la captura de la juventud inteligente hasta la jubilación o muerte del investigador" y para el cual la evaluación de la eficiencia del apoyo a sus proyectos de investigación no se restrinja a si se publicó o no un artículo "pues la calidad del trabajo

\section{Pérez-Tamayo R.}

Basic science and applied science.

Salud Publica Mex 2001;43:368-372.

The English version of this paper

is available at: http://www.insp.mx/salud/index.html

\section{Abstract}

A lecture was presented by the author at the Democratic Opinion Forum on Health Teaching and Research, organized by Mexico's National Health Institutes Coordinating Office, at National Cardiology Institute "Ignacio Chavez", where he presented a critical review of the conventional classification of basic and applied science, as well as his personal view on health science teaching and research. According to the author, "well-conducted science" is that "generating reality-checked knowledge" and "mis-conducted science" is that "unproductive or producing 'just lies' and 'non-fundable'. To support his views, the author reviews utilitarian and pejorative definitions of science, as well as those of committed and pure science, useful and useless science, and practical and esoterical science, as synonyms of applied and basic science. He also asserts that, in Mexico, "this classification has been used in the past to justify federal funding cutbacks to basic science, allegedly because it is not targeted at solving 'national problems' or because it was not relevant to priorities set in a given six-year political administration period". Regarding health education and research, the author asserts that the current academic programs are inefficient and ineffective; his proposal to tackle these problems is to carry out a solid scientific study, conducted by a multidisciplinary team of experts, "to design the scientific researcher curricula from recruitment of intelligent young people to retirement or death". Performance assessment of researchers would not be restricted to publication of papers, since "the quality of scientific work and contribution to the development of science is not re-

Trabajo presentado en el Foro de Consulta Democrática de la Coordinación de los Institutos Nacionales de Salud sobre Enseñanza e Investigación en Salud, realizado el 27 de marzo de 200I, en el Instituto Nacional de Cardiología Ignacio Chávez, México, D.F.

(I) Profesor Emérito de la Universidad Nacional Autónoma de México, Miembro de El Colegio Nacional y de la Academia Mexicana de la Lengua. Departamento de Medicina Experimental, Facultad de Medicina, Universidad Nacional Autónoma de México.

Fecha de recibido: 30 de marzo de 200I - Fecha de aprobado: 5 de abril de 2001 Solicitud de sobretiros: Dr. Ruy Pérez Tamayo. Apartado Postal 7064I, Ciudad Universitaria, 4510 México, D.F., México. Correo electrónico: ruypt@hotmail.com 
científico y la contribución de un investigador al desarrollo de la ciencia no es nada más el número de sus publicaciones". El texto completo en inglés de este artículo está disponible en: http://www.insp.mx/salud/index.html

Palabras clave: ciencia; tecnología; enseñanza; investigación; México flected by the number of published papers". The English version of this paper is available at: http://www.insp.mx/salud/ index.html

Key words: science; technology; teaching; research; Mexico
$\mathbf{R}$ ecientemente, en reunión con los nuevos directivos del Consejo Nacional de Ciencia y Tecnología (Conacyt), y los miembros del Consejo Consultivo de Ciencias de la Presidencia de la República Mexicana, uno de los puntos que se discutieron (y en el que me complace decir que hubo acuerdo unánime) fue la necesidad de apoyar con igual interés a la ciencia básica y a la ciencia aplicada. De hecho, en su primera intervención, el ingeniero Parada habló de sus proyectos para establecer vínculos con distintas secretarías de estado y aumentar de esa manera la inversión en Ciencia y Tecnología (CyT), logrando que el sector científico y académico se interese en estudiar y, si es posible, proponer soluciones a problemas específicos que competen a las secretarías; también se refirió a conversaciones ya iniciadas con el sector empresarial y expresó su satisfacción ante la buena acogida de sus gestiones, señalando que habría incentivos fiscales para la inversión en CyT por parte de la iniciativa privada. También agregó que se identificarían áreas específicas dentro de la problemática nacional, en las que la CyT pudiera tener impacto, y que se estimularía a científicos y tecnólogos para que se interesaran en ellas. No las llamó "prioridades" porque es un hombre de buen gusto, pero fue lo único que le faltó. Y cuando la mayoría de los miembros del Consejo Consultivo de Ciencias estábamos empezando a deprimirnos, el ingeniero Parada dijo que también se apoyaría a la ciencia básica, que estaba profundamente convencido de su importancia, que sabía muy bien que sin ciencia básica nada de todo lo demás era posible, y otras cosas más por el estilo que hicieron que el sol brillara de nuevo.

Tácitamente se acepta que hay esas dos clases de ciencia, la aplicada y la básica, una cuyos resultados sirven para resolver problemas definidos, que casi siempre son el estímulo que desencadenó el proceso de investigación, y otra que produce información que nos hace conocer mejor un fenómeno pero que no tiene aplicación práctica inmediata. Esta clasificación utilitarista de la ciencia también se ha expresado usando otros términos, como ciencia "pura" y ciencia "comprometida" (que estuvo de moda a fines de la década de los 60, aunque lo contrario de pura no es comprometida sino impura, lo que parecía inconveniente a los revolucionarios del 68 pero resulta atractivo para los ecologistas de hoy); ciencia "útil" y ciencia "inútil", que además de revelar el origen utilitario del concepto disfrutó de popularidad en Inglaterra, donde alguna vez el famoso matemático Hardy brindó porque su ciencia "nunca sirviera para absolutamente nada"; ciencia "esotérica" y ciencia "práctica", que también subraya la interpretación pragmática de la clasificación y aplica un término peyorativo a la ciencia supuestamente despreocupada por el uso ulterior de sus resultados.

Ignoro el origen y la antigüedad de esta división utilitarista de la ciencia, pero es seguro que no se le ocurrió a ningún científico activo; más bien parece producto de la cerebración de algún economista desvelado. A ningún miembro del gremio de la ciencia se le ocurriría que el conocimiento, que es el único producto de la actividad científica, puede no servir para nada, por la sencilla razón de que es él quien siempre lo usa, en primer lugar y antes que todos sus instrumentos, sus telescopios y sus centrífugas. Para los científicos, el principal uso del conocimiento es la generación de más conocimiento, los nuevos datos permiten integrar hipótesis que sugieren predicciones y experimentos para ponerlas a prueba, y es en este proceso en el que surgen más hechos, en el que se hacen los descubrimientos; es como se produce el conocimiento científico. De manera que los científicos tenemos razón de sentirnos incómodos cuando se habla de ciencia "básica" y ciencia "aplicada". Toda la ciencia es aplicada porque todo el conocimiento sirve para algo, todo se aplica, en primer lugar, para hacer más ciencia; además, ciertos conocimientos pueden servir para otras cosas, como resolver problemas médicos, o de distribución del agua, o de la conservación de granos, etcétera. Es posible que el desvelado economista clasificador se haya basado en la visibilidad del uso 
del conocimiento para influir en circunstancias externas a la estructura de la ciencia, tomando a esas aplicaciones como su única utilidad, y que se haya dejado llevar por la frase de Francis Bacon, "knowledge is power", pensando que el poder al que se refería el sabio inglés era (naturalmente) el económico, pero los investigadores científicos profesionales y activos en laboratorios o en el campo tenemos otra visión, creo que más amplia y más generosa, sobre los objetivos y los resultados de nuestra actividad, y otro concepto menos limitado de sus aplicaciones.

En alguna discusión con un buen amigo, filósofo profesional, me decía que la clasificación de la ciencia en "básica" y "aplicada" es útil porque distingue el trabajo científico que sólo sirve para seguir acumulando conocimientos con base en los generados por esa actividad, del que además resulta en aplicaciones prácticas externas al desarrollo y crecimiento de la ciencia. Según su punto de vista, las dos clases de ciencia serían la "básica" y la "básica y aplicada"; mi amigo termina su argumento diciendo que al tercer tipo teóricamente posible de ciencia, la puramente "aplicada", ya no la llama ciencia sino tecnología. En cambio, otro buen amigo, también filósofo, está convencido de que la separación entre ciencia y tecnología, si bien pudo tener algún sentido y hasta cierta utilidad en el pasado, actualmente ya no se justifica porque las dos actividades se han entremezclado de manera tan íntima que ya son inseparables y ahora conviene referirlas como "tecnociencia". Finalmente, un tercer buen amigo, igualmente filósofo, me preguntó: “¿Se puede saber para qué sirve esa clasificación de la ciencia en 'básica' y 'aplicada'...?"

En ese momento no se me ocurrió una respuesta razonable, pero después pensé que lo único para lo que ha servido en el pasado entre nosotros es para justificar la reducción en el apoyo oficial a la primera, porque no estaba dirigida a resolver los "problemas nacionales" o porque no caía dentro de las "prioridades" establecidas en ese sexenio.

Puesto a clasificar a la ciencia, creo (y así lo he dicho y escrito en otras ocasiones) que la única clasificación útil es en relación con su calidad, en ciencia bien hecha y ciencia mal hecha. También creo que la bien hecha es la que genera conocimientos verificables sobre la realidad y es la que debería apoyarse, mientras que la mal hecha no produce nada (o puras mentiras) y no debe patrocinarse. Para distinguir entre las dos ciencias, la bien hecha y la mal hecha, cuando todavía se encuentran en proyecto, existen ya distintos criterios y diferentes experiencias en todo el mundo, incluyendo a México; no son perfectos y ade- más están excesivamente burocratizados, pero en la mayoría de los casos cumplen con el requerimiento básico de depender de juicios emitidos por comités de científicos experimentados, en lugar de estar en manos de funcionarios administrativos. Lo que se juzga es complejo: la originalidad de la pregunta, el diseño de la generación de datos y su pertinencia para contestarla, la experiencia previa de los investigadores, sus facilidades instaladas y su presupuesto, la formación de recursos humanos, entre otras cosas. Pero la diferencia fundamental entre los comités de hombres de ciencia y los administradores es que los primeros saben que la investigación científica que vale la pena es la exploración de lo desconocido, de la pregunta cuya respuesta realmente se ignora y por lo tanto no puede planearse, mientras los segundos no conciben que los resultados no sean anticipables y hasta cronológicamente predecibles. Naturalmente, hay proyectos de todas clases, desde los que se plantean como: "¿Y qué pasa si...? que generalmente identifican a campos de la ciencia en etapas iniciales de desarrollo, hasta los que definen su objetivo en forma específica, como: "Frecuencia de la amibiasis en profesores universitarios mayores de 70 años", que casi siempre son complemento de programas de investigación ya muy avanzados.

Pero en todos los proyectos científicos bien hechos existe un elemento adicional, algo que los del gremio conocemos muy bien, porque lo hemos experimentado con frecuencia, y que en cambio se escapa por completo a los funcionarios, administradores y burócratas, porque cuando lo encuentran tienden a obliterarlo como una anomalía o un defecto en el sistema, algo que no salió como debía, que no estaba planeado. Me refiero a la serendipia, ${ }^{\circledR}$ al resultado inesperado, a la sorpresa que al principio intriga y al final convence, porque no era lo que esperábamos pero con su novedad nos abre nuevas posibilidades, a veces tan distintas de nuestras predicciones iniciales pero al mismo tiempo tan atractivas que abandonamos nuestro propósito original para seguir la nueva senda que quién sabe a dónde nos lleve. Siempre me ha sorprendido que la experiencia de la serendipia, tan frecuente en la vida profesional de los investigadores científicos activos, haya recibido tan escasa atención en los escritos de filosofía de la ciencia; en cambio, me explico perfectamente que la serendipia no figure en ningún discurso, proyecto, organigrama, presupuesto, Plan Nacional, o campaña política nacional. Y es que la serendipia no se sujeta a estructuras predecibles: su naturaleza misma las excluye, no porque sea irracional, sino porque su razón de ser se basa en configuraciones que todavía 
no conocíamos cuando hicimos el proyecto de investigación y que sólo empiezan a revelarse a través de su inesperada presencia.

Hasta aquí he dicho solamente dos cosas, las dos bien conocidas: la primera, que la única clasificación aceptable de la ciencia es en bien hecha y mal hecha, y que debemos olvidarnos de la última; y la segunda, que los resultados de la ciencia bien hecha no son anticipables, porque ésta consiste en hacer preguntas sobre lo desconocido. Si los resultados de un proyecto de investigación son tan predecibles que hasta permiten su calendarización rigurosa por cuatrimestres dentro de un periodo fijo de 2 a 3 años -como lo exigen los formatos del Conacyt y de otras agencias aportadoras de recursos-, entonces ya casi no vale la pena llevarlo a cabo. La burocratización de la ciencia es su peor enemigo, porque pretende transformarla, de una aventura del pensamiento, en un contrato por obra determinada. Para los científicos, el laboratorio es un espacio de actividad esencialmente creativa, mientras que para los burócratas más bien se parece a un taller de reparación de automóviles. Acepto que es indispensable que los automóviles caminen y que haya talleres que los mantengan en funcionamiento, pero sostengo (en el sentido de Pereira, el admirable personaje de Tabucchi) que la ciencia es otra cosa.

Lo dicho hasta aquí debe servir como prólogo (demasiado largo, por cierto) a lo que deseo contribuir al tema de esta reunión, que es Enseñanza e Investigación en Salud. Interpreto que el interés de los organizadores ha sido estimular y recoger ideas generales y proyectos específicos para orientar y promover la investigación de los problemas de salud en nuestro país. Sospecho que la respuesta a su convocatoria no fue tumultuosa o siquiera excesiva, lo que era anticipable en vista de nuestra falta de experiencia en este tipo de ejercicios democráticos; la tradición política administrativa mexicana siempre había sido que cada nuevo sexenio, para iniciar sus mandatos, algunas autoridades (desde luego, no todas) presentaran a la ciudadanía sus programas de trabajo, programas que además estaban sujetos a todo tipo de cambios durante la vigencia de su gestión política. Tales programas eran el producto de las ideas del señor Secretario en turno o de una comisión formada ad hoc para redactarlos, pero siempre fieles a las directivas previamente expresadas por el señor Presidente. La democracia no es un don genético sino una forma adquirida de vida política, y como todo lo externo a nuestro genoma requiere aprendizaje, necesita de la experiencia acumulativa y progresiva de aprender de nuestros errores, cada vez más, a través del tiempo. Creo que este Foro es un síntoma de que estamos en el buen camino.
Tengo dos propuestas específicas para este Foro, una para la enseñanza y la otra para la investigación en salud. En relación con la enseñanza, creo que debemos reconocer que el esfuerzo que ha hecho México en las últimas décadas para establecer una comunidad científica de la versatilidad y el tamaño necesarios para que sus actividades influyan en el nivel y la calidad de vida de la ciudadanía, ha fracasado en gran parte. No que el gremio de los investigadores no haya crecido en los últimos 50 años, pero el crecimiento ha sido mucho más lento que el de la población del país, por lo que hoy somos todavía menos por cada 10000 habitantes que hace medio siglo. El reconocimiento del fracaso mencionado es apenas el primer paso; el siguiente es realizar un análisis crítico y riguroso de sus causas, que seguramente son múltiples y probablemente están distribuidas en todo el sistema. Los médicos sabemos muy bien que para instituir un tratamiento adecuado, lo indispensable es un diagnóstico integral correcto. La pregunta es ¿a qué se debe que el programa actual de formación de investigadores tenga una eficiencia tan baja? Naturalmente, todos tenemos opiniones al respecto, pero no son más que eso, opiniones. Lo que se necesita es un estudio científico crítico, realizado por un equipo de especialistas en distintas áreas de la sociología, de la educación, de la economía y otras disciplinas más, que sirva de base para formular un nuevo programa de formación de investigadores, para integrar la carrera de investigador científico que vaya desde la captura de la juventud inteligente hasta la jubilación o la muerte del investigador. Esto puede hacerse en poco tiempo y seguramente arrojará información sobre otros aspectos de la comunidad científica que también requieren actualización. Considero que el fracaso de nuestros mecanismos actuales de formación de científicos es una área de desastre y que corregirlo es una emergencia nacional.

Mi segunda propuesta se relaciona con el apoyo a la investigación en salud. Cualquiera que sea el esquema que se adopte para mejorar el actual, como ampliación del presupuesto, establecimiento de áreas preferenciales, participación de la iniciativa privada, etcétera, hay dos aspectos que, en mi opinión, deben cuidarse de manera muy especial: uno es la calidad de los proyectos que finalmente se apoyan y el otro es la medida de la eficiencia terminal de ese apoyo. Sobre el primero ya hice algunos comentarios, de manera que me limitaré a reiterar que esa es en verdad la única "prioridad" que un país tan pobre en investigación científica como México puede fijarse. Sería trágico que buenos proyectos, presentados por investigadores reconocidos por su capacidad y produc- 
tividad, que además incluyen alumnos en formación, no fueran apoyados porque no corresponden a áreas preseleccionadas usando cualquier tipo de criterios. Mi otro comentario es sobre la eficiencia terminal del apoyo a la investigación. En la actualidad se juzga casi exclusivamente por la publicación de un artículo, pasando por alto la realización misma del trabajo, la educación de investigadores jóvenes, la experiencia adquirida por el jefe del proyecto, la generación de nuevas ideas sobre el tema estudiado o sobre otros más o menos afines, etcétera. Esta reducción al mínimo de una experiencia tan prolongada y tan compleja proviene, en mi opinión, de la deformación progresiva de la evaluación del investigador científico introducida por los llamados "índices bibliométricos" hace ya más de 30 años por Eugene Garfield, el director del Institute for Scientific Information. De acuerdo con el mismo Garfield, esos "índices" (número de publicaciones, número de citas, impacto de la revista, etcétera) no deben usarse para evaluar el trabajo de científicos individuales, y tampoco deben usarse como los únicos datos para juzgar a grupos, instituciones, comunidades o países. Pero como los índices se desarrollaron (según el mismo Garfield) para poder evaluar un trabajo científico sin tener que leerlo, los administradores se apoderaron de ellos y los usaron precisamente para lo que su autor proclamaba que no servían. La reacción de las comunidades científicas no se hizo esperar: si la calidad del trabajo de un investigador se mide por el número de sus publicacio- nes por unidad de tiempo (digamos, al año), entonces aparecieron los artículos firmados por 15, 30 o más autores, la publicación de los mismos datos en diferentes revistas, los MPD (siglas de minimal publication data) para fraccionar un trabajo en dos o más, etcétera, otros signos todos de la fiebre del publish or perish, como los anglosajones le llaman a lo que en buen castellano se conoce como prostitución. Esta práctica es rutina en nuestro medio, y citaré dos ejemplos: a) el informe anual que los investigadores miembros del Sistema Nacional de Investigadores debemos someter al Conacyt solicita únicamente el número de publicaciones (no el título, no los resultados, no la revista donde aparecieron), simplemente el número; $b$ ) hace varios años, cuando solicité la contratación de un nuevo investigador para mi grupo, la primera pregunta que me hicieron fue: ¿cuántos trabajos ha publicado? Temo que si seguimos por este camino, la siguiente pregunta será: ¿cuánto pesan?

Mi punto de vista es que la evaluación de la eficiencia del apoyo a un proyecto de investigación no puede restringirse a si se publicó o no un artículo. La calidad del trabajo científico no es nada más eso, como la contribución de un investigador al desarrollo de la ciencia no es nada más el número de sus publicaciones. Tenemos que rescatar toda la riqueza y la versatilidad de nuestra profesión de la estrecha cárcel unidimensional en la que la han encerrado los administradores. 